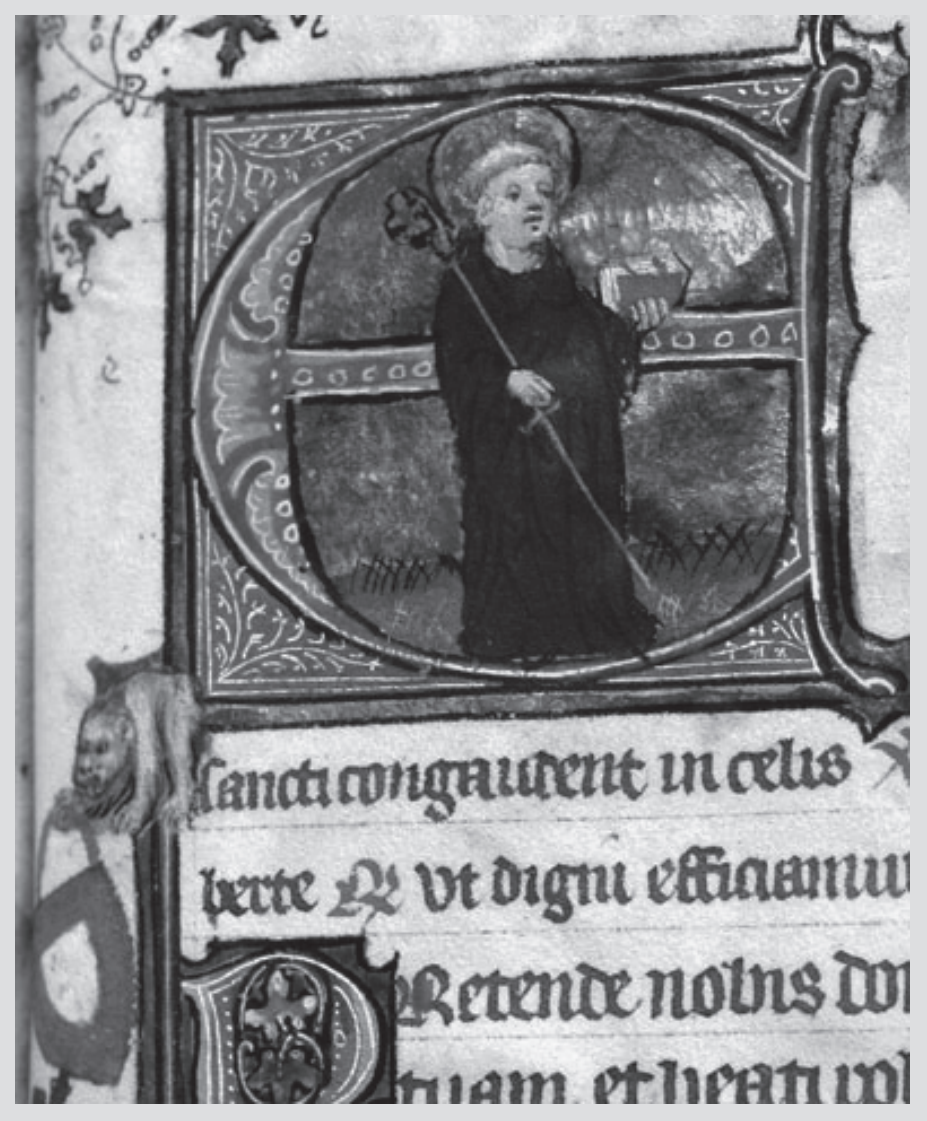

\author{
PUBLICATION \\ DU CENTRE EUROPEEN D'ETUDES \\ BOURGUIGNONNES (XIVe - XVIe s.) \\ $N^{\circ} 50-2010$
}

RENCONTRES D'AVIGNON

(17 au 20 septembre 2009)

«L'Église et la vie religieuse, des pays bourguignons à l'ancien royaume d'Arles (XIVe-XVe siècle)» 


\author{
ALAIN MARCHANDISSE \\ Maître de recherches au F.N.R.S. - Université de Liège \\ et \\ BERTRAND SCHNERB \\ Université Charles-de-Gaulle-Lille III
}

\title{
LE LIVRE DE PRIÈRE DE ROBERT ET MARGUERITE, SEIGNEUR ET DAME DE WAVRIN (FIN DU XIV ${ }^{\mathrm{e}}$ SIÈCLE)
}

L'étude de la piété et des dévotions de la noblesse de la fin du Moyen Âge ne semble pas, aujourd'hui, être l'une des grandes préoccupations des chercheurs ${ }^{1}$, sinon sous l'angle très spécifique de l'histoire de l'art et de l'histoire du livre ${ }^{2}$. Encore faut-il remarquer que dans ce type d'approche, le noble est essentiellement, sinon exclusivement, vu comme un commanditaire ou un bibliophile et non comme un laïc envisagé dans le cadre de sa pratique religieuse. C'est le cas des nombreuses

1 On peut toutefois citer P. CONTAMINE, La piété quotidienne dans la haute noblesse à la fin du Moyen Âge: l'exemple de Charles d'Orléans (1463-1465), dans Horizons marins, itinéraires spirituels ( $V^{e}-X V I I I^{e}$ siècles), t. 1, Mentalités et sociétés, éd. H. DuBoIs, J.-C. Hocquet, A. Vauchez, Paris, 1987, pp. 35-42 (article repris dans P. Contamine, De Jeanne d'Arc aux guerres d'Italie. Figures, images et problèmes du XV siècle, Orléans-Caen, 1994, pp. 205-212) ; A. Marchandisse, L'ordre de Saint-Antoine en Hainaut et L'homme à l'œillet de la Gemäldegalerie de Berlin. Quelques prolégomènes provisoires, dans Liber amicorum Raphaël de Smedt, t. 2, Artium Historia, éd. J. Vander Auwera, Louvain, 2001, pp. 117-131 ; B. SCHNERB, Piété et culture d'une noble dame au milieu du XV siècle : l'exemple de Marguerite de Bécourt, dame de Santes, dans Au cloître et dans le monde. Mélanges en l'honneur de Paulette L'Hermite-Leclercq, sous la dir. de P. Henriet et A.-M. Legras, Paris, 2000, pp. 235-245 ; ID., La piété et les dévotions de Philippe le Bon, duc de Bourgogne (1419-1467), dans Comptes rendus des Séances de l'Académie des Inscriptions et BellesLettres, 2005, pp. 1319-1344 ; ID., Les ducs de Bourgogne de la Maison de Valois et les frères mendiants : une approche documentaire, dans Économie et religion. L'expérience des ordres mendiants, XIII ${ }^{e} X V^{e}$ siècle, éd. N. Bériou et J. ChIfFoleAu, Lyon, 2009, pp. 233-279 ; ID., Die Frömmigkeit Karls des Kühnen, dans Burgundisch-Habsburgische Hofkultur 1450-1550. 16. Workshop des Arbeitskreises "Höfe des Hauses Österreich 》, 15 oct. 2009, sous presse.

2 Voir, par exemple, H. WiJsman, Gebonden Weelde. Productie van geïllustreerde handschriften en adellijk boekenbezit in de Bourgondische Nederlanden (1400-1550), Leyde, 2003 (éd. en anglais, Turnhout, 2010, sous presse) et Livres et lectures de femmes en Europe entre Moyen Age et Renaissance. Textes réunis par A.-M. Legaré, Turnhout, 2007. 
études qui ont été menées sur les livres d'heures et les livres de piété en général ${ }^{3}$, à quelques exceptions près ${ }^{4}$.

Certains de ces livres offrent pourtant des informations de première importance sur la pratique religieuse ; le caractère concret et direct de ces informations est d'autant plus marqué que l'identité du possesseur de l'ouvrage peut être établie et que le signe d'une utilisation courante peut, lui-même, être constaté. C'est le cas du manuscrit de la Bibliothèque nationale de France coté Nouvelle acquisition française 4412.

Ce manuscrit de 200 sur $135 \mathrm{~mm}$ est volumineux, puisqu'il ne compte pas moins de 365 feuillets. Il était assurément, à l'origine, plus important car, si l'on suit la foliotation originale, on constate que 162 feuillets, au moins, sont perdus.

Ce livre a déjà intéressé les chercheurs, à commencer par Jean Sonet qui l'a analysé dans son Répertoire d'incipit de prières en ancien français et y a relevé l'existence d'une dizaine de textes originaux ${ }^{5}$. Il a plus récemment attiré l'attention de Dominique Vanwijnsberghe qui, avec l'approche d'un historien d'art, en a fait une étude poussée lui permettant d'identifier l'origine du manuscrit ainsi que l'atelier dont il est sorti et d'émettre une hypothèse concernant le premier commanditaire et possesseur ${ }^{6}$.

Penchons-nous d'abord sur l'origine géographique. L'étude du calendrier qui figure au début du manuscrit ${ }^{7}$ a conduit ceux qui l'ont étudié à conclure à une origine tournaisienne. On y trouve, en effet, la fête de saint Éleuthère, évêque de Tournai, le 20 février $^{8}$, et la fête de sa Translation (le 25 août) ${ }^{9}$, ainsi que la fête de la Dédicace de l'église de Tournai (le 9 mai ${ }^{10}$. Si l'on ajoute que l'étude du style des peintures ornant ce manuscrit a permis à Dominique Vanwijnsberghe d'en attribuer sans aucun doute possible la réalisation au peintre tournaisien Jean Semont, on ne peut que conclure que ce livre de prière a été réalisé à Tournai.

On peut aller plus loin en remarquant, toujours à propos du calendrier, que s'y reflète l'influence des frères mendiants et, singulièrement, des franciscains. On y relève en effet la fête de la Translation de saint François $(25 \text { mai })^{11}$, la fête de saint

3 Pour nous en tenir à un seul exemple : A. ChÂTELEt, L'âge d'or du manuscrit à peintures en France au temps de Charles VI, Dijon, 2000.

4 V. Leroquais, Un livre d'heures de Jean sans Peur, duc de Bourgogne (1404-1419), Paris, 1939.

5 J. SONET, Répertoire d'incipit de prières en ancien français, Genève, 1956, passim.

6 D. VAnWIJNsBerghe, Moult bons et notables. L'enluminure tournaisienne à l'époque de Robert Campin (1380-1430), Paris-Louvain-Dudley Ma, 2007, pp. 145-184, 336-341.

7 BnF, nouv. acq. fr. 4412 , ff. $2 \mathrm{v}^{\circ}-14 \mathrm{r}^{\circ}$.

8 Ibid., fol. 4 r.

9 Ibid., fol. 10 ro. Cette fête vient s'ajouter à celle de saint Louis de France.

10 Ibid., fol. $6 \mathrm{v}^{\mathrm{O}}$.

11 Ibid., fol. $7 \mathrm{r}^{\mathrm{o}}$. 
Antoine de Padoue, désigné comme saint Antone de l'ordene des freres meneurs, le 13 juin, et de son octave (le 20 juin) ${ }^{12}$, la fête de sainte Claire (12 août) ${ }^{13}$, la fête de saint Louis de Marseille (le 19 août) ${ }^{14}$, la fête des Plaies de saint François $(17 \text { septembre })^{15}$, la fête de la Translation de sainte Claire $(2 \text { octobre })^{16}$, la fête de saint François (4 octobre) et son octave (11 octobre $)^{17}$.

Ces informations indiquent donc un lien probable avec le couvent des franciscains de Tournai, cette fois non plus sur le plan de la production matérielle, mais de l'activité intellectuelle et spirituelle ${ }^{18}$.

Nos devanciers ont également pu identifier le commanditaire et possesseur de ce manuscrit ou, pour être plus exact, l'un des commanditaires et possesseurs. Ils se sont, pour cela, référés à deux miniatures, l'une figurant au fol. $310 \mathrm{r}^{\mathrm{o}}$ et l'autre au fol. $362 \mathrm{r}^{\mathrm{o}}$. Intéressons-nous d'abord à la miniature du fol. 362 (ou CCCC IIII ${ }^{\mathrm{xx}}$ III $\mathrm{r}^{\mathrm{o}}$ de l'ancienne foliotation-dernier feuillet utilisé) : elle représente saint Robert de Molesmes, avec la robe noire des bénédictins, la crosse abbatiale dans une main et un manuscrit de sa règle dans l'autre (ill. 1). Le personnage est inscrit dans l'initiale E. On voit, à gauche un animal, sans doute un singe (ill. 2), tenant un écu sur lequel figurent des armoiries qui se lisent : «d'azur à l'écusson d'argent en abîme ». Ce sont les armes de la famille de Wavrin ${ }^{19}$. Cette miniature associe donc le prénom Robert aux armes de Wavrin.

La miniature du fol. 310 (CCCC XXI ro de l'ancienne foliotation - où se trouve un récit rimé en langue vulgaire intitulé La vie saint Jeorge) représente un gentilhomme portant, par-dessus son armure, une cotte armoriée aux armes de Wavrin devant, derrière et sur les manches. Ce personnage est à genoux, en prière devant saint Georges représenté à cheval (ill. 3).

Ces indices ainsi que le style de l'armement, qui est de la fin du XIv e siècle, renvoient donc à un Robert, seigneur de Wavrin, vivant dans les années 1380 1390, contemporain du Schisme, ce qui explique la présence, dans le manuscrit qui nous occupe, du texte d'une messe pour le union de le papalité2 ${ }^{20}$. À ces dates et à cette époque, il s'agit, sans doute possible, de Robert VII de Wavrin.

12 Ibid., ff. $7 \mathrm{v}^{0}$ et $8 \mathrm{r}^{\circ}$.

13 Ibid., fol. $9 \mathrm{v}^{\mathrm{o}}$.

14 Ibid., fol. $10 \mathrm{r}^{\circ}$.

15 Ibid., fol. $11 \mathrm{r}^{\mathrm{o}}$.

16 Ibid., fol. $11 \mathrm{v}^{\mathrm{o}}$.

17 Ibid.

18 Sur ce couvent franciscain fondé avant 1235, voir W. Simons, Bedelkloosters in het graafschap Vlaanderen, Bruges, 1987, pp. 54-56.

19 Voir l'armorial de la cour d'amour de 1401. C. Bozzolo et H. Loyau, La cour amoureuse, dite de Charles VI, t. 1, Paris, 1982, pp. 94-95, $\mathrm{n}^{\circ} 106$.

20 BnF, nouv. acq. fr. 4412, fol. $91 \mathrm{r}^{\mathrm{0}}$ (CC XXXI). 


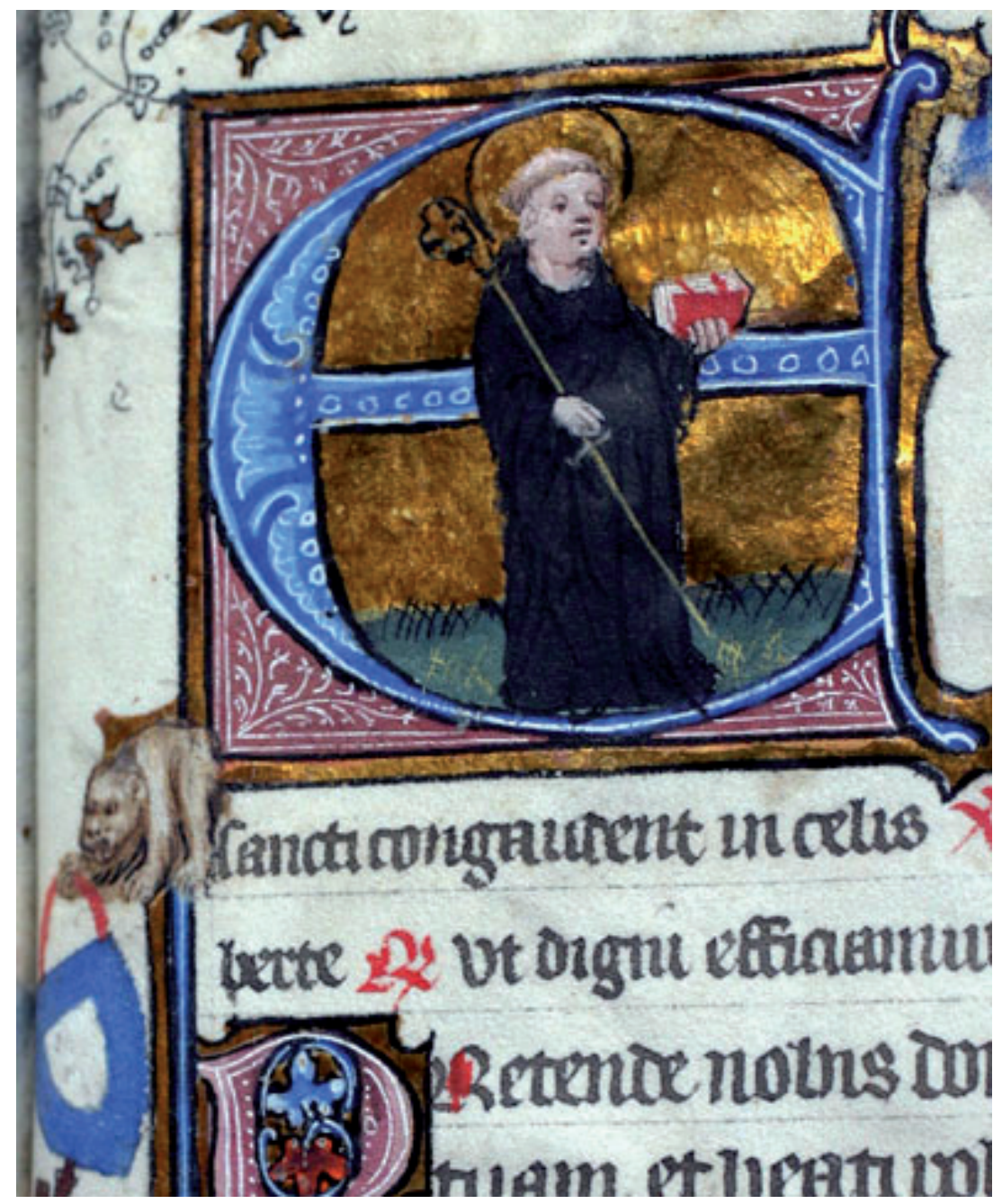

Ill. 1 : saint Robert de Molesmes, Livre de prière de Robert et Marguerite, seigneur et dame de Wavrin, PARIS, BnF, n. acq. fr. 4412, fol. 362 r $^{\circ}$ 


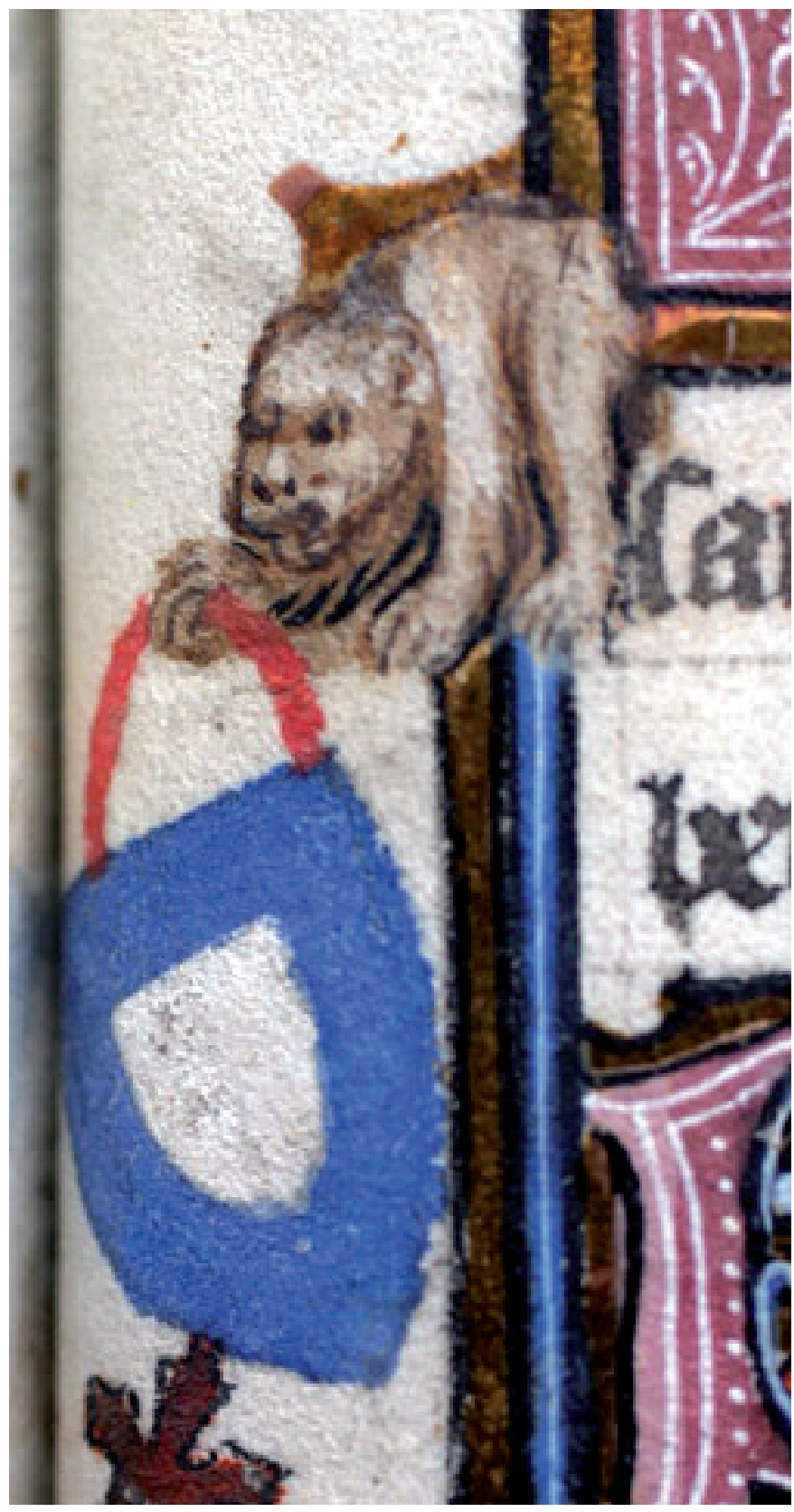

Ill. 2 : singe présentant un écu aux armoiries de Robert, seigneur de Wavrin, Livre de prière de Robert et Marguerite, seigneur et dame de Wavrin, PARIS, BnF, n. acq. fr. 4412 , fol. $362 \mathrm{r}^{\circ}$, détail 


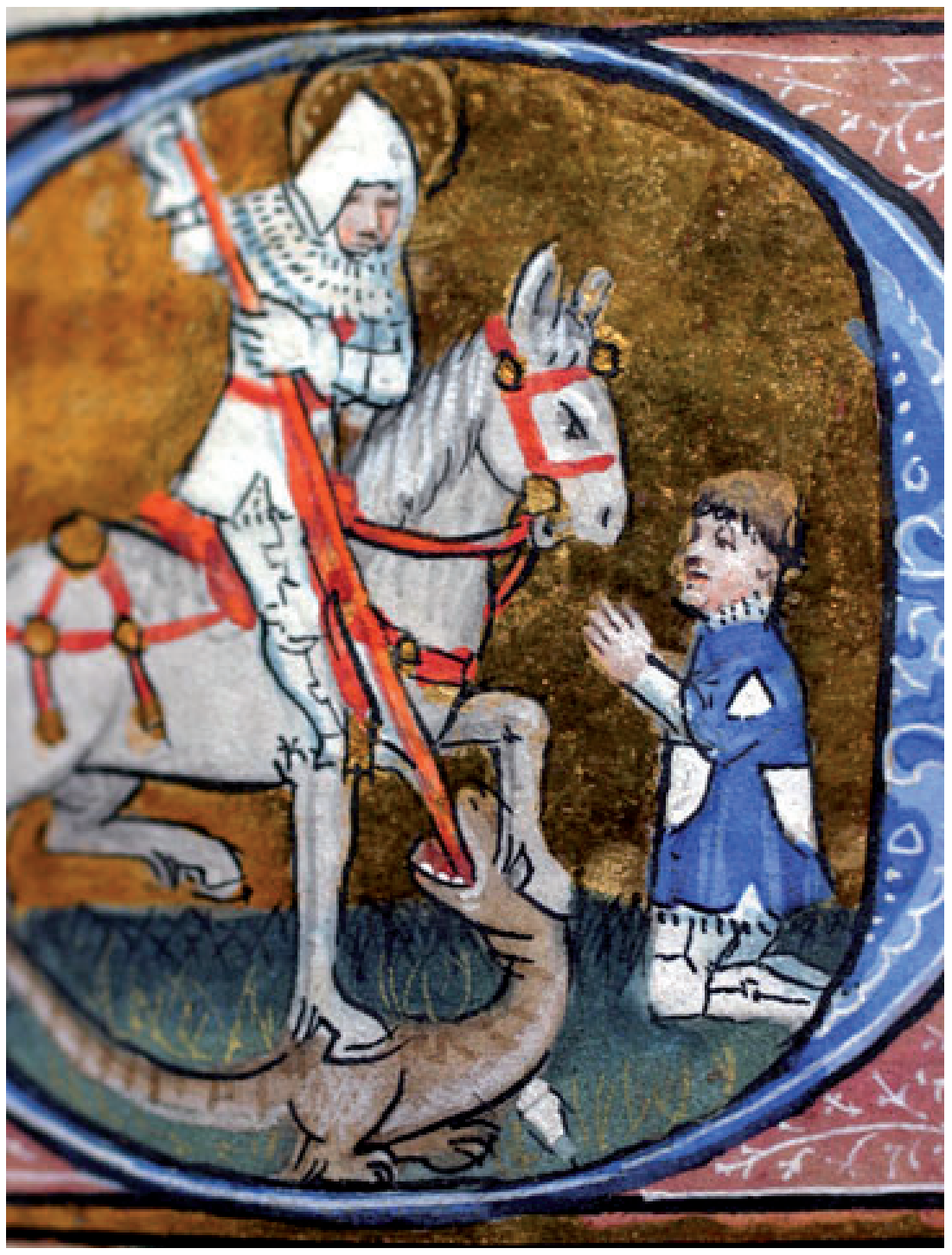

Ill. 3 : Robert VII, seigneur de Wavrin, en prière devant saint Georges, Livre de prière de Robert et Marguerite, seigneur et dame de Wavrin, PARIS, BnF, n. acq. fr. 4412, fol. $310 \mathrm{r}^{\circ}$ 


\section{Robert VII, seigneur de Wavrin}

Ce seigneur était issu d'une puissante famille possessionnée, depuis les XII et $\mathrm{XIII}^{\mathrm{e}}$ siècles, dans les comtés de Flandre et d'Artois ${ }^{21}$. La seigneurie de Wavrin ${ }^{22}$ était un fief tenu du comte de Flandre "à cause de sa Salle de Lille ${ }^{23}$ et le seigneur de Wavrin était, avec le châtelain de Lille, le seigneur de Comines et le seigneur de Cysoing, l'un des quatre seigneurs hauts-justiciers de la châtellenie. Ces seigneurs avaient d'importants privilèges et notamment celui de consentir à la levée des aides ${ }^{24}$. Par ailleurs, depuis la fin du XIV siècle, les seigneurs de Wavrin possédaient aussi, au comté d'Artois, les seigneuries hautes-justicières de Lillers ${ }^{25}$ et de Malannoy ${ }^{26}$, qui étaient tenues, en un seul fief, du comte d'Artois à cause de la Cour-le-Comte d'Arras ${ }^{27}$. À ce titre ils faisaient partie de la haute aristocratie artésienne et figuraient dans les premiers rangs des représentants de la noblesse aux États d'Artois ${ }^{28}$.

Robert VII († 1415) était le fils de Pierre de Wavrin et de Marie d'Arleux ${ }^{29}$. Pierre, cadet de famille, avait survécu à ses deux frères aînés Robert V († 1361) et Guillaume († 1365). Lorsque son neveu Robert VI, fils de Robert V, mourut sur

21 La meilleure étude sur la famille de Wavrin entre le $\mathrm{XI}^{\mathrm{e}}$ et le $\mathrm{XIV}^{\mathrm{e}}$ siècle se trouve dans R. JACOB, La minorité de Robert VI de Wavrin. Question de bail, garde et douaire dans la coutume d'Artois au XIV siècle d'après la jurisprudence du Parlement, Liège, 1978. On peut toutefois encore consulter avec profit F. Brassart, Une vieille généalogie de la Maison de Wavrin publiée avec des notes historiques et héraldiques sur les sénéchaux et connétables de Flandre, Douai, 1877.

22 Nord, arr. Lille, cant. Haubourdin.

23 Hommage reçu par le comte Louis de Male le 22 mars 1383 (n. st.). Lille, Archives départementales du Nord (=ADN), B 4078 et F. Brassart, Une vieille généalogie, p. 37.

24 Le duc Jean sans Peur donna, le $1^{\text {er }}$ octobre 1414, des lettres patentes par lesquelles il déclarait reconnaître solennellement qu'il était dans l'obligation d'obtenir leur accord pour la levée des aides consenties par les États des châtellenies de Lille, Douai et Orchies. Ordonnances de Jean sans Peur, 1405-1419, éd. J.-M. Cauchies, Bruxelles, 2001, pp. 357-358, nº 219 et p. 418 , $\mathrm{n}^{\circ} 254$.

25 Pas-de-Calais, arr. Béthune, ch.-l. cant.

26 Pas-de-Calais, arr. Béthune, cant. Norrent-Fontes, com. Bourecq.

27 Hommage reçu par le comte Louis de Male le 22 mars 1383 (n. st.). ADN, B 4078 et F. BRASSART, Une vieille généalogie, p. 37. Voir aussi R. JACOB, La minorité de Robert VI, p. $151 \mathrm{n} .2$.

28 Voir la «liste des prélats, chapitres, bonnes villes et nobles d'Artois » établie lors de la conclusion de la paix d'Arras au début de 1415 : le nom de Robert VII, seigneur de Wavrin, vient en troisième position dans la liste des nobles, après Jean, seigneur de Croy, et Jean de Croy, son fils. L. Mirot, Autour de la paix d'Arras (1414-1415), dans Bibliothèque de l'École des Chartes, t. 75, 1914, pp. 253-327 (cf. p. 325). Voir aussi C. Hirschauer, Les États d'Artois de leurs origines à l'occupation française, 1340-1640, t. 1, Paris-Bruxelles, 1923, pp. 36-37 et 40.

29 Robert VII avait une sœur, Jeanne de Wavrin, qui épousa Jean, seigneur de Rosimbos († 1415). F. Brassart, Une vieille généalogie, pp. 36-38 ; D. Schwennicke, Europäische Stammtafeln, nlle sér., t. 8, Marbourg, 1980, tabl. 23. 
le champ de bataille de Rozebeke, le 27 novembre 1382, sans laisser d'héritier légitime, il recueillit les seigneuries de Wavrin, Lillers et Malannoy ${ }^{30}$. Il fit hommage pour ces trois fiefs au comte de Flandre Louis de Male au mois de mars 1383 et les laissa aussitôt à son fils Robert VII en avancement d'hoirie ${ }^{31}$.

À ce moment, Robert VII, qui porta dès lors les titres de seigneurs de Wavrin, Lillers et Malannoy ${ }^{32}$, était déjà un chevalier et un homme marié. Il avait en effet épousé, à une date que nous ignorons, Marguerite, bâtarde de Flandre, fille illégitime du comte Louis ${ }^{33}$. Cette Marguerite, dame de Wavrin, est mentionnée dans le testament de Louis de Male daté du 29 janvier 1384 par lequel le comte recommandait certains de ses bâtards à ses exécuteurs testamentaires ${ }^{34}$. Elle mourut en 1389, après avoir donné deux enfants à son mari : un garçon, Robert VIII, et une fille Béatrice de Wavrin ${ }^{35}$. Robert VII, pour sa part, se remaria en août 1390 à Jeanne de Grincourt ${ }^{36}$, qui lui donna une fille, Jeanne de Wavrin ${ }^{37}$.

30 R. JACOB, La minorité de Robert VI, pp. 150-151.

31 Le 22 mars 1383 (n. st.) à Lille, le comte de Flandre déclare avoir reçu l'hommage que Pierre de Wavrin, écuyer, lui fit pour trois fiefs, à savoir le fief de Lillers et de Malannoy et le chastellenie d'icelle, descendant de nostre chastel et court d'Arras. Item, de le terre de Wavrin et des appartenances, descendant de nostre Salle de Lille. Item, d'un autre fief descendant de nostre chastel de Buvry [Beuvry]. Lesquelx fiefs, ainsi qu'il dist, li appartiennent et sont escheuz par la mort de feu le seigneur de Wavrin, darrain trespassé, nepveu audit Pierre de Wavrin, comme son plus apparant et prochain hoir. Le même jour, le comte reçut l'hommage pour ces trois fiefs de Robert de Wavrin, filz de Pierre de Wavrin, esquelz ledit Robert, a la poursuite, requeste et du consentement d'icelli Pierre de Wavrin, son pere, pour certaines et justes causes, et en avanchement d'oyrie, fu, par luy, en nostre presence et par nostre attroy, come aisné fil et plus prochain hoir d'icelli Pierre de Wavrin, dehuement adhiretez. F. Brassart, Une vieille généalogie, pp. 36-37.

32 Dans un acte du 15 juillet 1397, le duc de Bourgogne informe le bailli d'Aire qu'il a donné quittance à Robert, seigneur de Wavrin, de Lillers et de Malannoy, pour une somme de $145 \mathrm{fr}$. d'or en quoy il povoit estre tenus pour quint denier et autrement a cause de l'achat par luy fait de XL livres de rente parisis sur la terre de Malannoy. ADN, B 1029.

33 Louis de Male avait assigné une rente annuelle de 700 1. p. à sa fille bâtarde à l'occasion de son mariage. P. De Lichtervelde, Les bâtards de Louis de Male, dans Handelingen van het Genootschap voor Geschiedenis, gesticht onder de Benaming «Société d'Émulation 》 te Brugge, t. 78, 1935, pp. 48-58 (cf. pp. 50-51).

$34[\ldots]$ par especial le Haze, Loys, dit le Frison, Hannekin, dit sans terre, Marguerite, dame de Wavrin, et la nonnain de Peteghem (Marguerite, abbesse de Peteghem). ADN, B 455.

35 F. BRASSART, Une vieille généalogie, p. 38 ; R. ЈАCOB, La minorité de Robert VI, pp. 151-152 et tableau généalogique II. Robert VIII épousa Jeanne de Créquy. D. Schwennicke, Europäische Stammtafeln, t. 8, tabl. 23.

36 Par mandement donné le 3 août 1390, le duc Philippe le Hardi quitte le seigneur de Wavrin du droit de quint qu'il aurait dû payer pour s'être devestis et deshiretés d'une rente annuelle de 8001.10 s. p. pour le douaire de Jeanne de Grincourt, pour entretenir le traictié du mariage qui se fait entre lui et ladite demoiselle. F. Brassart, Une vieille généalogie, pp. 38-39. A propos du douaire de cette dame, voir une lettre adressée par le procureur d'Artois aux gens de la Chambre des comptes de Lille en date du 31 mars 1419 (n. st.). ADN, B 17 625. Grincourtlès-Pas, Pas-de-Calais, arr. Arras, cant. Pas-en-Artois.

37 R. JАСОВ, La minorité de Robert VI, pp. 151-152 et tableau généalogique II. 


\section{Une carrière nobiliaire}

La carrière de Robert VII de Wavrin est conforme à celle d'un représentant de la noblesse d'armes de son temps. Sa présence dans les armées royales ou princières est attestée à partir des années 1380 : ainsi en 1385, il prit part à l'expédition d'Écosse avec l'amiral Jean de Vienne ${ }^{38}$. On le retrouve, l'année suivante, sous la bannière du duc de Bourgogne, alors que se préparait une invasion de l'Angleterre qui n'eut jamais lieu ${ }^{39}$.

Ce service d'armes fut suivi d'une intégration à la cour de Bourgogne et, singulièrement, à l'entourage du comte de Nevers, futur duc Jean sans Peur, auprès duquel Robert VII, seigneur de Wavrin, se trouve fréquemment à partir des années 1398-1400 ${ }^{40}$. Ensuite, en 1405, lorsque Jean devint comte de Flandre et d'Artois, Robert fut retenu en tant que conseiller et chambellan ${ }^{41}$, sa fille, Béatrice de Wavrin, figurant, pour sa part, parmi les demoiselles de l'hôtel de la duchesse de Bourgogne Marguerite de Bavière en $1406^{42}$.

38 Le 27 avril 1385, à Arras, Robert de Wavrin, chevalier bachelier, donne quittance au trésorier des guerres du roi pour 2001 . t. à lui versées en prêt sur ses gages et ceux d'un autre chevalier bachelier et de trois écuyers de sa chambre et sur son estat d'un franc d'or par mois pour chaque homme de sa compagnie. Ses gages étaient à desservir en ceste presente armee que le roy nostre dit seigneur met presentement sus pour le passage d'Escoce, en la compaignie et soubz le gouvernement de messire Jehan de Vienne, admiral de France, chief et cappitaine de ladite armee. Le 8 mai suivant, il donne une autre quittance de gages datée de L'Écluse. $\mathrm{BnF}$, Clairambault, $110, \mathrm{n}^{\text {os }} 8613$ et 8615 ; F. Brassart, Une vieille généalogie, p. 39 ; H. Terrier de Loray, Jean de Vienne, amiral de France. 1341-1396, Paris, 1878, p. CXXV.

39 Entre le $1^{\text {er }}$ sept. et le 30 nov. 1386, Robert est à la tête d'une compagnie forte de 3 chevaliers bacheliers (lui non compris) et 20 écuyers (compte du trésorier des guerres Guillaume d'Enfernet, 1386-1387-BnF, ms. fr. 7858, fol. $267 \mathrm{r}^{\mathrm{o}}$ ).

40 L. Mirot, Jean sans Peur de 1398 à 1405 d'après les comptes de sa chambre aux deniers, dans Annuaire-Bulletin de la Société de l'Histoire de France, 1938, pp. 129-245 (cf. pp. 168, 169 et 189).

41 Il figure déjà dans l'entourage de ce duc lorsque ce dernier fait sa Joyeuse Entrée à Gand en avril 1405 (il est mentionné parmi les personnalités présentes dans l'acte par lequel Jean sans Peur s'engage par serment à respecter les privilèges de la ville). Ordonnances de Jean sans Peur, p. 9, ${ }^{\circ} 5$. Ensuite, par lettres patentes du duc en date du 16 juillet 1405, Robert, seigneur de Wavrin, chevalier, conseiller et chambellan, est retenu, moyennant une somme de $80 \mathrm{fr}$. versée à la fin de chaque mois lorsqu'il sert devers mondit seigneur en son hostel. DiJon, Archives départementales de la Côte-d'Or (= ADCO), B 1547, fol. 77 v $^{\circ}$ et B 1554, fol. $63 \mathrm{v}^{\circ}$. Le 15 avril 1410, Jean sans Peur donne l'autorisation à son amé et feal chevalier, conseillier et chambellan, messire Robiert, seigneur de Wavrin, de Lillers et de Malannoy, d'établir une confrérie d'arbalétriers à Wavrin. Ordonnances de Jean sans Peur, pp. 181-182, $\mathrm{n}^{\circ} 123$. À noter qu'il figure dans la célèbre Cour d'amour, dite de Charles VI, en tant que chambellan du roi de France. C. Bozzolo et H. Loyau, La cour amoureuse, t. 1, pp. 94-95, $\left.\mathrm{n}^{\circ} 106\right)$.

42 ADN, B 1878, fol. $172 \mathrm{r}^{\circ}$. 
Parallèlement à son service de cour, le seigneur de Wavrin servit le duc Jean sans Peur dans ses guerres, participant notamment à la défense de la Flandre contre les Anglais en $1405^{43}$, au « voyage de Liège » et à la bataille d'Othée en $1408^{44}$, ainsi qu'aux expéditions de France de 1411 et $1412^{45}$. Par ailleurs, il fut investi de nombreuses missions diplomatiques ${ }^{46}$. C'est ainsi qu'il fut envoyé auprès du comte de Saint-Pol, alors en Brabant, en $1406^{47}$, auprès du roi de France en $1408^{48}$, auprès du comte de Hainaut en 1410 et $1413^{49}$, auprès du duc de Berry en $1411^{50}$.

43 En mai 1405, il est reçu à montre lorsque des troupes sont levées pour défendre la Flandre contre les Anglais, après la bataille de Marcq. Il est alors mentionné comme chevalier banneret et est à la tête d'une compagnie comptant un chevalier bachelier (Jean de Rosimbos, son beau-frère), 16 écuyers et 14 archers.

44 Il est mentionné à cette occasion par Enguerrand de Monstrelet, Chronique, éd. L. DouËt D’ArcQ, t. 1, Paris, 1857, p. 372 ; Chronique du règne de Jean de Bavière, éd. S. Balau et É. FaIron, t. 1, Bruxelles, 1913, p. 198 ; La Bataille du Liège, éd. P. F. X. De Ram, Documents relatifs aux troubles du pays de Liège, sous les princes-évêques Louis de Bourbon et Jean de Horne, 1455-1505, Bruxelles, 1844, p. 307, en attendant une nouvelle éd. commentée par B. Schnerb et A. Marchandisse. Il est reçu à montre le 15 septembre 1408 à la tête d'une compagnie comptant un autre chevalier banneret (Guillaume, seigneur de Bours), 5 chevaliers bacheliers, 61 écuyers et 78 archers et arbalétriers à cheval (ACO, B 11771 J. De La Chauvelays, Les armées des trois premiers ducs de Bourgogne de la maison de Valois, dans Mémoires de l'Académie des Sciences, Arts et Belles-Lettres de Dijon. Partie des Lettres, Année 1880, 1881, p. 176). Après la campagne, le seigneur de Wavrin, conjointement avec le seigneur de Saint-Georges, le seigneur de Croy, Jacques de Courtiambles, Jean de Nielles, seigneur d'Olhain, et Guillaume, seigneur de Bonnières, reçoit 10000 écus « de l'argent venant du païs de Liege » (soit plus de 1600 écus par tête) par mandement du duc donné le 16 février 1409 (n. st.) (ACO, B 1558, fol. 69 ro).

45 À l'été et à l'automne 1411, il figure dans l'armée du duc de Bourgogne qui fait campagne en Picardie et dans la région de Paris. BnF, nouv. acq. fr. 20528, p. 196 ; En juin 1412 (pour le voyage de Bourges), il reçoit des gages pour lui, deux chevaliers, dix-sept écuyers et quinze archers de sa compagnie servant sous le duc de Bourgogne. BnF, Clairambault, 110, $\mathrm{n}^{\circ} 8617$; F. Brassart, Une vieille généalogie, p. 40.

46 À noter toutefois que son nom ne figure pas dans C. De Borchgrave, Diplomaten en diplomatie onder hertog Jan zonder Vrees. Impact op de Vlaamse politieke situatie, 2 vol., Kortrijk-Heule et Bruxelles, 1992.

47 Sacent tout que je, Robert, seigneur de Wavrin, conseillier et chambellan de monseigneur le duc de Bourgoingne, conte de Flandres, d'Artois et de Bourgoingne, congnois avoir eu et receu de Jehan Saquespee, commis par mondit seigneur a recevoir un aide nagaire ottroyé a mondit seigneur le duc en sadicte conté d'Artois, ressors et enclavemens, pour les grans affaires qu'il a eux depuis la desconfiture de Merq, la grant assamblee de gens d'armes qu'il lui a convenu faire pour le proufit et utilité de ce royaume, et pour le joyeux advenement a la seignourie d'icelle conté, pour avoir allé, par l'ordonnance de mondit seigneur, ou païs de Brabant devers monseigneur de Saint Pol, qui lors y estoit, pour lui requerir, aveuc moy messire Loys du Quesnoy, qu'il voulsist acorder a mondit seigneur le duc ledit aide en sadicte conté de Saint Pol, ouquel voyage je vagay par cinq jours commenchans le XXe jour d'avril derrain passé, pour chascun jour IIII l. X s. t., sont XXII l. Xs. tournois. De laquelle somme de XXII l.X s. t. je me tiens a contens et en quitte ledit receveur et tous autres. Tesmoing mon seel et saing manuel chi mis le XII jour de jullet l'an mil CCCC et six. ADN, B 1879, nº 53077.

48 ADCO, B 1576, fol. $211 \mathrm{v}^{\circ}$.

49 ADCO, B 1560, fol. $143 \mathrm{r}^{\circ}$ et B 1576, fol. $236 \mathrm{v}^{\circ}$.

50 ADCO, B 1570, fol. $94 \mathrm{r}^{\circ}$. 
Robert VII, seigneur de Wavrin, et son fils Robert (VIII) tombèrent tous deux sur le champ de bataille d'Azincourt le 25 octobre 1415 et furent inhumés en l'abbatiale de Ham-en-Artois ${ }^{51}$.

\section{La piété du seigneur (et de la dame) de Wavrin}

Robert VII, seigneur de Wavrin, avait sans doute choisi l'abbaye de Ham comme lieu de sépulture, car, depuis sa fondation, elle était liée étroitement aux seigneurs de Lillers ${ }^{52}$. Sa tombe, et celle de son unique fils légitime ${ }^{53}$, fut placée dans la chapelle Saint-Benoît : le caveau aménagé dans cette chapelle, où Agnès de Witternesse, dame de Wavrin, morte en 1343, s'était déjà fait enterrer, semble avoir joué le rôle de caveau familial ${ }^{54}$; la chapelle fut reconstruite et agrandie sous l'abbatiat de Jacques de Rosimbos, abbé de Ham de 1438 à 1460 ou 1461, neveu de Robert VII de Wavrin 55 .

L'attachement du seigneur de Wavrin à l'abbaye de Ham serait le seul indice concernant sa piété si nous ne possédions son livre de prière. Celui-ci, comme il a été dit plus haut, montre en premier lieu une influence franciscaine qui s'explique, peut-être, par l'appartenance du confesseur des commanditaires à l'ordre des frères mineurs ${ }^{56}$. En second lieu le manuscrit, par sa décoration, indique une dévotion à saint Robert de Molesmes (fête le 29 avril), patron de baptême d'un nombre non négligeable de membres de la famille de Wavrin, car le prénom Robert, qui était celui du fondateur de la dynastie seigneuriale, fut non seulement porté par huit

51 Voir l'épitaphe qui figurait sur leur tombe aujourd'hui disparue : Cy gisent messire Robert, seigneur de Wavrin, de Lillers et de Malannoy, chevalier, et messire Robert de Wavrin, son fils et heritier, chevalier, et trespasserent ensemble a la bataille d'Azincourt qui fut l'an mil IIII et XV, le XXV ${ }^{\text {me }}$ jour du mois d'octobre. Priez Dieu pour leurs ames et de tous les trespassez. Et feist cest lame faire madame Beatrix, dame de Wavrin et de Lillers et de Malannoy, fille et sour des ditz trespassez. Priez Dieu pour eux. Texte édité dans : Épigraphie ou recueil des inscriptions du département du Nord ou du diocèse de Cambrai, éd. T. LeURIDAn, t. 3, Lille, 1905, p. 850. Voir aussi H. Bernard, L'abbaye d'Ham-en-Artois. Recherches d'archéologie médiévale et classique, 2 vol., Arras, 1998, spécialement t. 1, pp. 89-92.

52 L'abbaye fut fondée en 1079 à la demande d'Enguerrand de Lillers et de sa femme Emma, sur un terrain par eux donné à l'abbaye de Charroux. Ibid., t. 1, p. 34.

53 Robert VII eut, comme chacun sait, des bâtards dont le plus connu est le chroniqueur et bibliophile Jean, bâtard de Wavrin. Voir, en dernier lieu, A. MARChANDisSE, Jean de Wavrin, un chroniqueur entre Bourgogne et Angleterre, et ses homologues bourguignons face à la guerre des Deux Roses, dans Littérature et culture historiques à la cour de Bourgogne. Actes des rencontres internationales de Dunkerque (octobre 2005), éd. J. DevauX et A. MarchandisSE, Le Moyen Âge, t. 112, 2006, pp. 507-527.

54 H. Bernard, L'abbaye d'Ham-en-Artois, t. 1, pp. 89-90.

55 Ibid., t. 1, p. 163. Jacques de Rosimbos était le fils de Jean de Rosimbos et de Jeanne de Wavrin, sœur de Robert VII. Cf. supra n. 28.

56 Sur l'influence des frères mendiants dans les milieux de cour, voir Könige, Landesherren und Bettelorden. Konflikt und Kooperation in West- und Mitteleuropa bis zum Frühen Neuzeit, sous la dir. de D. Berg, Werl, 1998. 
représentants de la branche aînée entre la fin du XII ${ }^{\mathrm{e}}$ siècle et 1415 , mais encore par trois Wavrin de la branche cadette des seigneurs de Saint-Venant, dont le plus connu fut Robert III, maréchal de France en $1340^{57}$.

À cette dévotion à la fois personnelle et dynastique, s'ajoute une dévotion à saint Georges qui revêt, elle aussi, une signification personnelle, puisque Robert de Wavrin s'est fait représenter en prière devant l'image du saint en tête d'un feuillet où a été copiée une Vie saint Jeorge; mais elle renvoie aussi à une réalité collective et sociale, puisque le saint chevalier est, comme on le sait, l'une des grandes figures des dévotions militaires et nobiliaires ${ }^{58}$.

Saint Georges, image parfaite du miles Christi, avant d'être « tropeophore » et « draconoctone », est d'abord un martyr qui « prend sa part de souffrance en bon soldat du Christ », comme l'écrit saint Paul ${ }^{59}$. Par ailleurs sa fonction d'intercesseur s'enrichit de celle d'un modèle dont le combat légendaire contre le dragon revêt une puissante signification allégorique ${ }^{60}$. Cette double fonction d'intercesseur et de modèle de combattant chrétien est clairement exprimée dans l'image représentant le seigneur de Wavrin, tout armé, en prière devant le saint, lui-même en armure.

L'identification de saint Georges en tant que saint martyr apparaît, quant à elle, non sous forme d'image, mais sous forme de texte: en effet, la représentation iconographique au folio 310, accompagne, comme nous l'avons dit, une courte pièce rimée intitulée La vie saint Jeorge, qui est en fait, essentiellement, un récit de son martyre (sa passion), récit qui est d'ailleurs, notons-le, complètement indépendant de celui de la Légende dorée de Jacques de Voragine ${ }^{61}$ et d'autres Vies connues du saint-chevalier ${ }^{62}$.

Cette courte Vie saint Jeorge précède immédiatement dans le livre de prière, une Vie sainte Marguerite beaucoup plus développée, puisqu'elle couvre les folios 311 à 321 . Il ne fait guère de doute que la présence de ce long texte - sans relation, lui non plus avec la Légende dorée ${ }^{63}$ - est un indice du rôle que Marguerite de Flandre,

57 F. BRASSART, Une vieille généalogie, passim et R. JACOB, La minorité de Robert VI de Wavrin, tableau généalogique II.

58 K. Pollems, Art. Georg (hl., Märtyrer), dans Lexikon des Mittelalters, éd. broch., t. 4, Munich, 2003, col. 1273-1275 ; S. Braunfels Esche, Sankt Georg. Legende, Verehrung, Symbol, Munich, 1976.

$592 \operatorname{Tm} 2,3$

60 S. Braunfels et E. Lucchesi Palli, Art. Georg, dans Lexikon der christlichen Ikonographie, t. 4, Rome, 1972, col. 365-390 ; A. Boureau, Saint Georges et le dragon, dans Formes médiévales du conte merveilleux, sous la dir. de J. Berlioz, C. Brémond, C. Velay-Vallantin, Paris, 1989, pp. 21-29 ; G. Didi-Huberman, R. Garbetta, M. Morgaine, Saint Georges et le dragon. Versions d'une légende, Paris, 1994.

61 BnF, nouv. acq. fr. 4412, ff. $310 \mathrm{r}^{\circ}-\mathrm{v}^{\circ}$. Jacques de Voragine, La Légende dorée, éd. et trad. J.-B. M. Roze et H. SAvon, 2 vol., Paris, 1967, I, p. 296-301.

62 Voir Deux versions de la vie de saint Georges, éd. Y. Gullcher, Paris, 2001.

63 Jacques de Voragine, La Légende dorée, I, pp. 452-455. 
dame de Wavrin, première épouse de Robert VII, a joué en tant que commanditaire et possesseur du manuscrit ${ }^{64}$. L'usage du nom de Marguerite comme nom de baptême et la dévotion à la sainte sont à rattacher à des usages bien attestés dans la famille comtale de Flandre depuis Marguerite de Constantinople (1202-1280) et plus spécialement au temps de Louis de Male, dont la mère (Marguerite de France), l'épouse (Marguerite de Brabant), la fille légitime (Marguerite de Male) et trois bâtardes ${ }^{65}$ portaient ce prénom.

Ce livre de Robert et Marguerite, seigneur et dame de Wavrin, nous renseigne enfin sur la pratique quotidienne de la prière, comme on peut le voir en étudiant sa structure interne.

\section{Morale, fins dernières et sacrements}

Le manuscrit s'ouvre sur une prière adressée à la sainte Face qui devait être prononcée devant une image de la Véronique. La foliotation ancienne montre que ce texte, qui a été copié au folio $1 \mathrm{r}^{\mathrm{o}}$ et $\mathrm{v}^{\mathrm{o}}$, était bien en tête du livre. Le texte qui suit cette oraison latine précise : De l'orison dessus dite doit on cascun jour saluer le Vironicle venant des pardons de Rome, en jun, devotement et on y acquert mil jours de pardon ${ }^{66}$. Il s'agissait donc d'une prière quotidienne et matinale. Il est probable qu'une image mobile, aujourd'hui perdue, rapportée du pèlerinage romain, ait été fixée à l'une des toutes premières pages du manuscrit, conformément à ce que Denis Bruna a pu décrire à propos des livres d'heures ${ }^{67}$.

Vient ensuite le calendrier, que nous avons déjà évoqué68, suivi du Dit des trois morts et des trois vifs ${ }^{69}$. La version copiée ici ne correspond à aucun des cinq

64 Il n'est donc pas trop audacieux d'avancer que la composition de ce livre de prière est antérieure à 1389, année de la mort de Marguerite, dame de Wavrin. Il est probable que cette dernière ait été le commanditaire originel et que Robert VII ait, après la mort de son épouse, personnalisé le manuscrit en faisant ajouter sur un feuillet libre une courte vie de saint Georges et, après le dernier feuillet utilisé, un suffrage à Robert de Molesmes. Ajoutons que dans le texte de certaines prières, des adjectifs ou participes se rapportant à l'orant sont au féminin : je viens vers vous pour i estre sanee $\left(\mathrm{BnF}\right.$, nouv. acq. fr. 4412, ff. $\left.56 \mathrm{r}^{\mathrm{o}}-\mathrm{CXIII}\right)$; je suy morte en mes peciés (fol. $56 \mathrm{v}^{\circ}$ ).

65 Marguerite, dame de Wavrin, Marguerite, abbesse de Peteghem, Marguerite de Flandre, épouse de Siger de Gand. P. DE Lichtervelde, Les bâtards de Louis de Male, pp. 51-52.

$66 \mathrm{BnF}$, nouv. acq. fr. 4412, fol. $1 \mathrm{v}^{\mathrm{o}}$.

67 D. Bruna, Témoins de dévotions dans les livres d'heures à la fin du Moyen Âge, dans Revue Mabillon, nlle sér., t. 9 (t. 70), 1998, pp. 127-161 (cf. pp. 130-132 pour les véroniques).

68 BnF, nouv. acq. fr. 4412 , ff. $2 \mathrm{v}^{\mathrm{o}}-14 \mathrm{r}^{\mathrm{0}}$.

69 Ibid., ff. $16 \mathrm{r}^{\mathrm{o}}-23 \mathrm{r}^{\mathrm{o}}\left(\mathrm{XXII} \mathrm{r}^{\mathrm{o}}-\mathrm{XXIX} \mathrm{r}^{\mathrm{o}}\right)$. Sur ce thème, voir : S. GliXelli, Les cinq poèmes des trois morts et des trois vifs, Paris, 1914 ; C. KIENNING, Le double décomposé. Rencontres des vivants et des morts à la fin du Moyen Âge, dans Annales. ÉSC, t. 50, 1995, pp. 1157-1190; W. Rotzler, Die Begegnung der drei Lebenden und der drei Toten, ein Beitrag zur Forschung über die mittelalterlichen Vergänglichkeitsdarstellungen, Winterthur, 1961; C. BASCETTA, Art. Trois morts et trois vifs (Légende des), dans Dictionnaire des Lettres françaises, sous la dir. de G. Hasehohr et M. ZinK, Paris, 1992, pp. 1453-1454. Sur la traduction iconographique du thème, voir en dernier lieu : Vifs nous sommes... Morts nous serons. La rencontre des trois morts et des trois vifs dans la peinture murale en France, Vendôme, 2004. 
poèmes de la nomenclature de Stefan Glixelli, mais semble une œuvre originale construite, en partie, par des emprunts aux poèmes I (celui de Baudouin de Condé), IV et $\mathrm{V}$ de cette nomenclature ${ }^{70}$. Si on le compare avec les poèmes édités, on peut estimer que ce texte est amputé de soixante à soixante-dix vers, en raison de la perte de plusieurs feuillets ${ }^{71}$.

À la suite (mais originellement séparée du Dit des trois morts et des trois vifs par neuf feuillets) vient une série de sept quatrains contenant des sentences morales rimées, dont voici un exemple ${ }^{72}$ :

Que plus te sourquides ${ }^{73}$ et plès,

Plus t'esvuides et en peril te mès.

S'aultrui vois bon, passe l'en bien,

Laisse l'aultrui mal, punis le tien.

La rubrique qui suit s'intitule Chi s'enssievent pluiseurs choses par lesquelles ont peut resister contre pechié et acquerir bonnes virtus ${ }^{74}$. Ce titre introduit une liste des $V$ sens de nature, des dix commandements, des VII vertus contre les VII pechiés morteulz, des XII articles de la foy contenus ou Credo, des VII dons du saint Esperit.

Une description imagée des tourments d'enfer vient ensuite :

Il y a fu, li quelz est si ardans que se toutes la mers couroit par dessus, point ne seroit estains. Du quel fu li ardeurs sourmonte le fu materiel en tel maniere que le fu materiel sourmonte le fu paint en une paroit. Et chis fus d'ynfer ne luit point et se il luist che n'est point a la consolation des malvais, mais pour che que il voient leurs tourmens. Et sy a froidure intollerable de laquelle il est escript: se une montaigne plaine de feu estoit mise dedens, elle seroit muee en glace. De ches deux paines est il escript que en ycellui lieu sera pleurs et estrainture de dens. Li fumee du fu esmeut les pleurs de yeulz et la froidure esmeut le estrainture des dens. Et se y sont les vers immorteulz, est a sçavoir, serpens, culeuvres, crapaus, dragons orribles de veue et de sifflement, lesquelz vivent en flamme comme les poissons en l'iauwe. Et si y a pueur si intollerable que se toutes les choses qui sont en che monde estoient muees en caroingnes, elles ne rendroient mie si grand puour. Et se y sont les ames batues des dyables ainsi que les martiaus freans sur le fer. Et la sont les tenebres palpables et

70 S. Glixelli, Les cinq poèmes, pp. 53-63, 83-91 et 92-110.

71 Les ff. XVI ro à XXI v ${ }^{\circ}$ manquent, mais étant donné que l'on compte environ 20 vers par page, il est raisonnable de penser que le texte du Dit des trois morts et des trois vifs commençait au fol. XIX.

72 BnF, nouv. acq. fr. 4412, fol. $24 \mathrm{r}^{\mathrm{o}}$ (XXXIX).

73 Se sourcuidier: $\mathrm{s}$ 'enorgueillir.

74 Ibid., ff. 25 ro-27 $\mathrm{r}^{\circ}$ (XL-XLII). 
menniables. Et se y est la confusion des pechiés, car entour les pecheurs tout leur pechiet apparront et se ne se porront muchier. Et se y est la vision des dyables les quelz il verront gectans estinchelles et si ne se porront muchier. Et si sont les liiens de fu, desquelz tout li membre des pecheurs seront estraint et si aront toudis faim et soif et se n'aront que boire ne que mengier, et morront toudis sans jamais cesser. Ches tourmens aront et aultres pluiseurs si grans que nulz ne les porroit dire ne penser sans jamais avoir joie. Et par especial il seront dolent de che qu'il seront privés de la vision de Dieu et de sa glore a laquelle ils ne porront jamais recouvrer ${ }^{75}$.

Ce texte est suivi de trois courts traités : le premier sur l'art de bien mourir commençant par : En vis meurt qui aprins ne l'a ; apreng a morir, si saras vivre, quar bien vivre ja ne sara qui aprins a morir n' ara $^{76}$; le deuxième sur la communion ${ }^{77}$; le troisième sur la confession ${ }^{78}$. Ces trois thèmes, qui ne sont pas sans évoquer la pastorale mendiante, se rattachent en tout état de cause aux préoccupations d'un confesseur.

Il est possible de constater que du fol. 16 au fol. 46, c'est-à-dire du Dit des trois morts et des trois vifs jusqu'à la collection de petits traités dont nous venons de parler, le concepteur du manuscrit a voulu constituer un premier ensemble de textes didactiques centré sur les questions primordiales de la morale, des fins dernières et des deux sacrements qui doivent être au cœur de la vie du fidèle. Ensuite, commence la partie du livre qui est proprement consacrée à la pratique de la prière ${ }^{79}$.

\section{La prière au quotidien}

Cette partie s'ouvre sur les heures de Notre Dame ainsy que on les doit dire depuis le premier samedi del Advent jusques a la veille du Noel ${ }^{80}$. Puis viennent des prières destinées à accompagner la vie sacramentelle : une oraison à prononcer avant la confession, une autre à dire après s'être confessé ${ }^{81}$ et une oraison à dire avant la communion; celle-ci attachée au récit d'une apparition du Christ à saint Augustin et qui y fait explicitement référence :

75 Ibid., ff. 31 ro-32 ro. Sur les peines de l'enfer, voir: J. FrAPPIER, Châtiments infernaux et peur du diable d'après quelques textes français $d u$ XIII et $d u$ XIV siècle, dans Cahiers de l'Association internationale des Études françaises, t. 3, 1953, pp. 87-96; J. Delumeau, Le péché et la peur. La culpabilisation en Occident, XIII ${ }^{e}-X V I I I^{e}$ siècle, Paris, 1983, pp. 416-426.

76 BnF, nouv. acq. fr. 4412, ff. $32 \mathrm{r}^{\mathrm{o}}-39 \mathrm{v}^{\mathrm{o}}\left(\mathrm{LV}-\mathrm{LXII} \mathrm{v}^{\mathrm{o}}\right)$.

77 Ibid., ff. $39 \mathrm{v}^{\mathrm{o}}-44 \mathrm{r}^{\mathrm{o}}$ (LXII vo-LXVII).

78 Ibid., ff. $44 \mathrm{r}^{\mathrm{0}}-46 \mathrm{r}^{\circ}$ (LXVII-LXIX).

79 La prière au Moyen Âge, Sénéfiance, t. 10, 1981 ; N. Bériou, J. Berlioz, J. Longère, Prier au Moyen Age, Turnhout, 1991.

80 BnF, nouv. acq. fr. 4412 , ff. 47 ro $-54 \mathrm{r}^{\mathrm{o}}$ (CI-CVIII).

81 Ibid., ff. $54 \mathrm{r}^{\mathrm{0}}-\mathrm{V}^{\mathrm{o}}\left(\mathrm{CVIII}-\mathrm{CVIII} \mathrm{v}^{\circ}\right)$. 
Biaus sires Dieus quy ainsy parlas a saint Augustin, je te prie, sire plain de pité et de misericorde, que quand je prendray ton saint corps que tu m'otroies qu'il me viegne a autel profit comme il fist a saint Augustin ; sy me veullies muer et croistre et conforter et manoir en toy en desirier de toy amer; et sy soiez tous temps avec moy, que n'aie desirier que de toy servir et a toy parvenir et ta pure face veir, quy tant par est doulce et clere que li angle qui sont plus cler que le sollayl sont tout enluminé de luy; il le voyent toudis et com plus le regardent et plus le desirent a veoir, tant est elle pure et clere. Sire, engrange ta grace en moy en desirier de toy servir, que a toy puisse parvenir et ta pure face veir. Sire, je ne sui mie digne que je prenge ton saint cors en moy ne que tu viengnez en moi, mais ce me reconforte que tu dis que des pecheurs as merci qui a toy retornent. Or, aiiés, sire, merchi de moy, car, sire, pour la grant pité de toy et nient pour merite qui soit en moy, serai ge hardié de ton saint cors recevoir en moy. Or vieng dont, sire, manoir en moy. Si me conforte en toy, si te combat pour moy encontre mes anemis, sy me conduis en paradis et me fay vivre ou chiel avec toy. Quod nobis concedat ille qui vivis et regnat Deus per omnia secula seculorum. Amen ${ }^{82}$.

On trouve après ce texte un autre groupe de prières de communion : une première à dire devant le corps Nostre Seigneur Jhesu Crist quand on le veult recevoir, une deuxième à dire avant de communier, puis les graces apres communier ${ }^{83}$.

Les prières suivantes sont conçues pour rythmer la vie du fidèle au quotidien : d'abord les heures du saint sacrement à dire tous les jours, les grâces à réciter « après dîner » et « après souper », puis les prières majeures : Pater noster. Ave Maria. Credo. Benedicite: Dominus, nos et ea que sumus... Ave salus mundi. Confiteor. Misereatur nostri, omnipotens Deus, avec cette mention spéciale :

Quand on est esveillié au matin, le premiere cose que on doit dire et aussy au nuit le derraine cose que on doit dire che doit estre le patrenostre quar c'est le plus belle et le plus souveraine orison de toutes les autres ${ }^{84}$.

Une collection de prières particulières est fournie, dans laquelle le fidèle peut puiser pour scander les différents moments de sa journée ${ }^{85}$ : une recommandation à Dieu quand on est assis dans son lit pour se lever au matin ou se coucher au soir ; une louange à prononcer quand on s'éveille ; deux prières à dire au matin ; au matin, toujours, doit on dire le Euvangille saint Jehan ${ }^{86}$ et puis saluer son bon angele et saint Christofle et puis dire ceste psalme tout au long : Deus in nomine tuo salvum

82 Ibid., ff. $55 \mathrm{r}^{\mathrm{0}-56} \mathrm{r}^{\mathrm{0}}$ (CXII-CXIII).

83 Ibid., ff. $57 \mathrm{v}^{0}-59 \mathrm{r}^{\mathrm{0}}$ (CXIIII v $\left.\mathrm{v}^{\circ}-\mathrm{CVXI}\right)$.

84 Ibid., ff. $83 \mathrm{r}^{0}-84 \mathrm{v}^{0}(\mathrm{CC}$ XXIII-CC XXIIII vo $)$.

85 Ibid., ff. $84 \mathrm{v}^{0}-88$ ro ${ }^{\circ}$ (CC XXIIII vo_CC XXVIII).

86 Probablement les premiers versets : In principio erat Verbum... (Jn 1,1). 
me $\mathrm{fac}^{87}$, car c'est bonne cose contre toute temptation; d'autres oraisons encore peuvent être dites au matin, au soir, quand on va se coucher ou au milieu de la nuit : Et au nuit on doit dire le hymne de Veni creator etc. et puis ceste orison: Deus qui corda fidelium, et saluer son bon angele et dire le Euvangile saint Jehan et saluer les IIII euvangeliste des orisons Huic camere etc. ou aultres orisons selonc le devotion, et puis dire son Confiteor et son Misereatur et se [sic] Pater noster tout au lonc comme est dit, et avoec les beneïçons comme dessus est dit.

Une autre série de prières doit accompagner le fidèle se rendant à l'église ${ }^{88}$ : en alant au moustier, il doit réciter: Vias tuas, Domine, demonstra michi et semitas tuas edoce me. Deduc me, Domine, in via tua et ingredia in veritatem tuam. Letetur cor meum ut timeat nomen tuum. En entrant dans l'église, il dit: Introibo in domum tuam. Adorabo ad templum sanctum tuum et confitebor nomini tuo. Une prière doit être prononcée en présence de l'hostie consacrée, une autre devant la croix, une enfin en prenant l'eau bénite (Aqua benedicta sit mihi salus et vita).

Cet ensemble de prières est suivi par des messes, certaines célébrées dans des intentions particulières sur lesquelles nous allons revenir, précèdent les messes de la Trinité, des Morts, des Apôtres, du Saint-Esprit, de la Croix, de Notre-Dame et du Saint-Sacrement ${ }^{89}$. Puis, suivant un enchaînement logique, sont énumérées les pensées, les prières et les attitudes du fidèle laïque pendant la messe ${ }^{90}$ : quand la cloche sonne ; lors de l'élévation de l'hostie ; lors de l'élévation du calice ; en présence de l'hostie consacrée ; après l'élévation ; lorsque le prêtre va communier (Sire, je ne sui mie digne que tu entres desous men toit, mais tant seulement di te parole et me ame sera salvee); enfin, à la fin de la messe.

Comme pour l'une des prières de communion, précédée d'un récit miraculeux mettant en scène saint Augustin, l'une de celles qui peuvent être dites lors de l'élévation (une succession de cinq salutations) est présentée comme dictée par le Christ à un clergeon anonyme :

Il fu un jones clers qui disoit a le elevation du corpz Nostre Signeur les $V$ gaude Nostre Dame. Advint une fois qu'i les disoit devant l'ymage Nostre Dame par grand devotion, et le ymage de Nostre Signeur que Nostre Dame tenoit li dist : "Bien plaist a me mere que ainsi le salues, mais pourquoy ne me salues tu aussi ? " Li clerçons li respondi que volentiers le feroit s'il sçavoit de quoy qui ly pleust, et Nostre Signeur li bailla ces $V$ Ave qui s'ensievent et li dist que bien li plairoit que de che par devotion le saluast a le elevation ou apres.

87 Ps 54.

88 BnF, nouv. acq. fr. 4412 , ff. $88 \mathrm{r}^{0}-\mathrm{V}^{0}\left(\mathrm{CC} X X V I I I r^{0}-\mathrm{V}^{0}\right)$.

89 Ibid., ff. $89 \mathrm{r}^{\mathrm{0}}-122 \mathrm{v}^{\mathrm{0}}\left(\mathrm{CC}\right.$ XXIX-CC LXV v $\left.{ }^{\circ}\right)$.

90 Ibid., ff. $123 \mathrm{r}^{\mathrm{o}}-152 \mathrm{v}^{\mathrm{o}}\left(\mathrm{CC} \mathrm{LXV}-\mathrm{CC} \mathrm{IIII}^{\mathrm{xx}} \mathrm{XV} \mathrm{V}^{\mathrm{o}}\right)$. 
Ce premier grand ensemble de prières qui doit accompagner le fidèle du matin jusqu'au soir, précède un second ensemble où sont regroupées les prières à la Vierge et aux saints.

Le manuscrit renferme un important ensemble de prières à la Vierge en français, suivi de variations sur le thème de la salutation angélique et de prières mariales en latin $^{91}$. Viennent ensuite une collection moins cohérente que les précédentes de prières propitiatoires, sur lesquels nous reviendrons, de nouvelles prières mariales, et de prières pour les trépassés et les âmes du purgatoire ${ }^{92}$. L'efficacité de ces dernières est prouvée par le témoignage des âmes délivrées.

Se aucuns voet faire cest service qui s'ensieut par L et I jours pour aucune ame, elle seroit delivree de quelcunques paine elle souffreroit en espurgatoire. Che esprouva une sainte nonne en le delivranche de sen frere ki fu occis, car quant elle eut fait che service, les frers s'aparu a li et dicist k'il estoit delivrés par ces priieres des paines et des tormens ke il devoit souffrir jusques au jour del jugement ${ }^{93}$.

C'est ensuite qu'a été intégrée la Vie de saint Georges, déjà évoquée, suivie de la Vie de sainte Marguerite. Viennent ensuite les heures de saint Louis de Marseille, les heures de saint François et les heures de sainte Catherine. Enfin, l'ensemble s'achève sur les suffrages pour les fêtes solennelles et pour les saints qui figurent au calendrier ${ }^{94}$.

\section{Pourquoi prie-t-on ?}

Le livre de prière de Robert et Marguerite de Wavrin est un précieux témoignage pour approcher la pratique de la prière des fidèles laïques. Lorsqu'on en étudie la substance, on constate, dans les intentions, une combinaison étroite de dévotion et d'utilitarisme, les vertus attribuées à la prière n'étant pas seulement d'ordre spirituel, mais aussi d'ordre matériel et, parfois même, divinatoire, thaumaturgique et magique.

Il apparaît d'abord que l'auteur de cette collection de prières, qui était lui-même un clerc, et peut-être le confesseur franciscain du seigneur et de la dame de Wavrin, a proposé à ses pénitents, des textes et des intentions conformes à l'enseignement de l'Église : prières et sacrements, qui sont ici intimement liés, visent à demander et à obtenir la grâce et une guérison spirituelle ; cette idée est bien exprimée dans une oraison préparant à la communion :

91 Ibid., ff. $155 \mathrm{r}^{0}-170 \mathrm{v}^{0}\left(\mathrm{CCC}-\mathrm{CCC}\right.$ XVI v $\left.{ }^{\circ}\right)$.

92 Ibid., ff. $177 \mathrm{r}^{\mathrm{o}-}-309 \mathrm{v}^{\mathrm{o}}\left(\mathrm{CCC}\right.$ XXIII-CCCC LXX v $\left.{ }^{\circ}\right)$.

93 Ibid., fol. $285 \mathrm{r}^{\circ}$ (CCCC XLVI).

94 Ibid., ff. 310 ro $-362 \mathrm{r}^{\mathrm{o}}$ (CCCC LXXI-Vc XXV). 
Sire, vous estez la vie de l'ame et je suy morte en mes peciés et mes negligences. Venés en moy si visités l'ame de mon corps qui ne puet vivre sans vous.

Les images temporelles viennent rendre plus concrète l'idée d'une grâce salvatrice :

Biaus sirez Dieus, vous estez mirez et medecins. Sire, je vieng a vous pour $i$ estre sanee. Sanés moy, sirez, sanés moy, car vous estes ma loenge. Sire, je suy obscurce par les tenebres de mes peciéz, mais je vieng a vous pour ce que vous estes lumiere. Purgiés moy mon cuer de [la] clarté de vostre grace ${ }^{95}$.

L'image du corps malade guéri par un Dieu médecin est prolongée par celle du château assiégé par l'ennemi, dont l'Eucharistie est l'approvisionnement et la garnison ; le fidèle ici est comme un vassal ou un capitaine s'adressant au seigneur qui lui a confié la garde d'une forteresse, mais doit lui porter secours en cas de péril :

Sire, je tieng cest castiel de vous et sy ne l'ay de coy garnir et sy ay moult d'anemis qui le veullent prendre. Sire, je vieng a vous que vous me garnissiés mon castiel de vous meisme ${ }^{96}$.

Les vertus spirituelles de la prière viennent aussi des pardons ou indulgences qui y sont attachés quand on les prononce dans les conditions requises : ainsi, la prière devant la Véronique obtient, est-il précisé, mille jours de pardon si elle est dite à jeun et devotement ${ }^{97}$; telle prière dite, à jeun, au moment de l'offertoire permet d'obtenir trois cens jours de vray pardon, mais qu'elle soit dite par grand devocion et repentance de ses pechyés ${ }^{98}$.

On peut noter que le concepteur du manuscrit a le plus souvent mentionné que les pardons étaient accordés par l'autorité pontificale et a identifié le pape à l'origine de leur octroi :

Papes Beneois XII del ordre de Citiaulz, composa ceste orison qui s'ensieut et donna a touz confes et contris disant par devotion a le messe en le presence du corps Nostre Seigneur pour cascune fois que on le dira o tant de jours de pardon que Nostre Seigneur est [sic] de plaiis le venredi benoit, qui furent en nombre de deux mile $I^{c}$ et $X X V I I^{99}[\ldots]$.

95 Ibid., fol. $56 \mathrm{r}^{\mathrm{o}}$ (CXIII).

96 Ibid., fol. $56 \mathrm{v}^{0}$.

97 Ibid., fol. 1 ro.

98 Ibid., fol. $139 \mathrm{r}^{\mathrm{o}}\left(\mathrm{CC}\right.$ IIII $\left.{ }^{\mathrm{xx}} \mathrm{I}\right)$.

99 Ibid., ff. $141 \mathrm{r}^{\mathrm{o}}-\mathrm{V}^{\mathrm{o}}\left(\mathrm{CC}\right.$ IIII ${ }^{\mathrm{xx}}$ III et $\left.\mathrm{v}^{\mathrm{o}}\right)$. 
Li papes Jehans XXII a donnet a tous cheus et a toutes celles qui diront ceste orison, $X X X$ mil jours de pardon dont li $X$ mil sont pour les pechiés morteulz et li $X X^{m}$ sont pour les pechiés venieux ${ }^{100}[\ldots]$.

Bonifasses papes $V I^{e}$ [sic], a le priere de Philippe, roy de Franche ${ }^{101}$, donna atous devotement disant ceste orison qui s'ensieut, $I^{m}$ ans de indulgence de pardon si le dist a heure de elevation du corpz Jhesu Crist ou quand on dist Agnus ou a le fraction du corpz Jhesu Crist ${ }^{102}$ [...].

Si certaines intentions de prière, plus temporelles que spirituelles, sont tournées vers la recherche du bien commun de la Chrétienté (la fin du Schisme ou la paix ${ }^{103}$ ), on constate que beaucoup d'autres ont une nette coloration propitiatoire. Certains textes sont même présentés comme ayant une destination précise pour répondre à des cas spéciaux : ainsi en est-il des psaumes attribués à saint Hilaire (auteur d'un psautier commenté ${ }^{104}$ ) pour prier Nostre Signeur des besoignes especiaulz : chacun des dix-huit textes de ce « psautier » répond à un cas précis : pour avoir rémission de ses péchés ; quand on est en tribulation ; pour faire une grande chose ; quand on se sent en la poesté du dyable; quand on est dans l'adversité du siècle ; quand on est en grande tristesse ; quand on se sent un trouble au cœur ; quand on veut que Dieu accomplisse notre désir ; quand on veut avoir Dieu débonnaire auprès de soi ; quand on est en grande nécessité ; quand on doit aller en un lieu qu'on redoute; quand on se lève de son lit le matin et qu'on est troublé par quelque chose ; quand on est dans l'adversité ; quand on doit plaider contre un homme puissant ; quand on doit combattre ou qu'un autre doit combattre pour nous ; quand on est en péché ; quand on veut faire le service ; quand on veut être sauf ${ }^{105}$.

La vertu de la répétition et de l'accumulation de certaines prières majeures est affirmée ; ainsi en est-il de l'Ave Maria :

Qui veult avoir Dieu debonnaire descendant a luy, sy die par X jours ensievant en juns et a genoulz devant l'autel mile Ave Maria, c'est cascun jour cent et apres les cent Ave Maria, dites III fois les orisons qui s'ensievent ${ }^{106}$ $[\ldots]$.

Il est vrai que le concepteur du manuscrit a orienté le fidèle vers une pratique qui, certes, répond à ses attentes, mais répond aussi à une conception plus spirituelle

100 Ibid., fol. $142 \mathrm{v}^{\mathrm{o}}\left(\mathrm{CC}\right.$ IIII $\left.{ }^{\mathrm{xx}} \mathrm{IIII} \mathrm{v}^{\mathrm{o}}\right)$.

101 Il s'agit évidemment de Boniface VIII (et non VI) et de Philippe IV.

102 Ibid., fol. $143 \mathrm{r}^{\mathrm{o}}\left(\mathrm{CC} \mathrm{IIII}^{\mathrm{xx}} \mathrm{V} \mathrm{r}^{\mathrm{o}}\right)$.

103 Ibid., ff. $187 \mathrm{r}^{0}-\mathrm{V}^{\mathrm{o}}\left(\right.$ CCC XXX III $\left.\mathrm{r}^{\circ}-\mathrm{V}^{\circ}\right)$.

104 Hilaire de Poitiers, Commentaires sur les Psaumes, éd. et tr. P. Decourtieux et J. Doignon, t. 1, Paris, 2008.

105 BnF, nouv. acq. fr. 4412, ff. $197 \mathrm{r}^{\circ}-199 \mathrm{r}^{\circ}$ (CCC XLIII-CCC XLV).

106 Ibid., ff. $195 \mathrm{v}^{\mathrm{o}}-196 \mathrm{v}^{\circ}(\mathrm{CCC}$ XL vo - CCC XLII vo $)$. 
que purement mécanique de la prière : ainsi en est-il des vingt-cinq Pater noster à prononcer vingt-cinq jours de suite pour obtenir une faveur de Dieu, car l'efficacité de la démarche est conditionnée par le fait que le requérant se soit confessé et qu'il soit en bon estat (entendons un bon état moral et spirituel); par ailleurs, l'exercice ouvre sur une méditation centrée sur les vingt-cinq souffrances du Christ au jour de sa Passion :

Qui dist ces $X X V$ paternostres cascun jour par XXV jours ensievant devant le crucefix en le ramembrance des XXV souffrances quel chose que che soit que on demandera pour sen profit, sachiés que Dieus li fera, mes que on soit confes et en bon estat et soit cascun jour en I pourpos en faisant la requeste tous ces jours et par especial faiche sa requeste ou $X X V^{e}$ jour par bonne devotion ${ }^{107}$.

Il y a là les traces visibles d'une volonté de concilier l'utilitarisme et la piété que l'on retrouve aussi dans les prières efficaces pour éviter les inquiétudes et les tourments de la vie quotidienne. Notre manuscrit contient par exemple des oraisons à prononcer quand on chemine ou pour trouver à l'étape un bon osteil et qui sont à dire en l'onneur de Dieu, de Nostre Dame et de saint Juliien. Les prières pour se préserver des tempêtes, des orages et du tonnerre sont épaulées à un récit rapportant comment le Christ rassure un religieux terrorisé par le tonnerre et qui le prie avec ferveur, en lui disant : Je sui en la crois penés et vous, ceste crois tenés, le jour que vous le garderés, tonnoire ne fourdre ne doubterés, ne nul mal encombrement ne feu ne aige ne vent ${ }^{108}$.

Il n'est pas indifférent de noter que si certaines prières propitiatoires ont une portée générale, d'autres sont plus nettement en relation avec les préoccupations probables de Robert et Marguerite de Wavrin, nos deux commanditaires-possesseurs. Ainsi en va-t-il de l'orison le quelle on doit dire pour estre et demourer en bonne amour entre mari et femme 109 et d'une longue prière en forme de litanie pour ne pas subir de dommages ni être desconfit en bataille ${ }^{110}$. La vertu de cette dernière prière est telle qu'en détenir le texte sur soi est une garantie, à condition d'avoir le droit pour soi :

Quand on va en bataille, qui sur lui l'auera

Jamais, s'il a bon droit, desconfis ne sera ;

Li papes Innocens le dit et confirma

Quand il ot confremee, apres si l'envoya

Charlemaine de Franche quand il combatre ala

Encontre Sarrasins en la terre dela

Pour le foy soustenir, mais tant y en trouva

107 Ibid., ff. $196 \mathrm{v}^{0}-197 \mathrm{r}^{\mathrm{o}}$ (CCC XLII vo-CCC XLIII).

108 Ibid., fol. $178 \mathrm{r}^{\circ}$ (CCC XXXIIII).

109 Ibid., ff. $186 \mathrm{v}^{\circ}-187 \mathrm{r}^{\mathrm{o}}$ (CCC XXXII vo_CCC XXXIII).

110 Ibid., ff. $183 \mathrm{v}^{0}-186 \mathrm{r}^{\circ}$ (CCC XXIX vo-CCC XXXII). 
Crestiens fussent perdus, may Dieu si leur aida,

Que le nuit esclarchi et le jour alonga.

Sains Jeorges y vint qui moult leur conforta,

Quand furent desconfit; sains Jeorges s'en ala,

Au bon roy Charlemaine ceste orison laissa.

Nulz homs n'est desconfis le jour qu'il le dira

Et qui ne le scet lire sur lui le portera.

L'orison est moult belle, oés qui le dira. Amen.

La référence à la Chanson de Roland et au cycle de Charlemagne est un bel indice de la rencontre de la culture religieuse et de la culture profane ${ }^{111}$. L'intervention de saint Georges, qui se substitue ici à saint Gabriel et rappelle sa présence aux côtés des croisés lors de la prise de Jérusalem mentionnée par la Légende dorée ${ }^{112}$, renvoie à la dévotion du seigneur de Wavrin. Il faut du reste rapprocher cette prière et la Vie saint Jeorges intégrée à notre manuscrit, puisque celle-ci est aussi présentée comme ayant des vertus protectrices pour les combattants :

Qui en bataille sa passion ara escripte,

Desconfis ne sera,

Puis que droit auera

Contre l'autre partie.

Et Dieu en sa pitié nous tiegne en sa partie ${ }^{113}$.

Le texte de la prière devient un talisman, même si la condition liée au bon droit (Dieu ne donnant la victoire qu'aux justes) se rattache à une logique ordalique. Quoi qu'il en soit, on observe un glissement qui, du terrain propitiatoire, nous place sur un terrain thaumaturgique et magique. Le phénomène est d'autant plus net lorsqu'on s'intéresse aux vertus curatives des Écritures et de la prière : Le Euvangille qui s'enssieut [Évangile selon saint Luc] fait on dire par ung prestre en grand devotion sur son chief par IX jours et en jun pour estre gary de toutes manieres de fievre ${ }^{114}$. Mais le caractère quasi magique est plus marqué encore à propos du texte d'une prière dont il est dit: Se homs ou femme a mal a son chief, si porteche cest brief pendu a sa diestre orelle, il garira de ce mal. Et qui ne poet dormir si ait ce brief desous son chief et il s'endormira ${ }^{115}$.

La protection contre la « mort soudaine », qui est l'un des thèmes récurrents dans les intentions ${ }^{116}$, n'est pas, elle non plus, sans reposer sur les vertus prophylactiques

111 La Chanson de Roland, éd. et trad. J. BÉDIER, Paris, 1937.

112 Jacques de Voragine, La Légende dorée, I, p. 301.

113 BnF, nouv. acq. fr. 4412, fol. $310 \mathrm{v}^{\circ}$ (CC LXXI vo $)$.

114 Ibid., ff. 204 v $^{\circ}-205$ r $^{\circ}$ (CCC L vo-CCC LI).

115 Ibid., ff. $193 \mathrm{v}^{\mathrm{o}}-195 \mathrm{v}^{\mathrm{o}}(\mathrm{CCC}$ XXXIX vo $-\mathrm{CCC}$ XL vo $)$.

116 Voir, par exemple, ibid., ff. $70 \mathrm{r}^{\mathrm{o}-74} \mathrm{v}^{\mathrm{o}}$ (C IIII ${ }^{\mathrm{xx}} \mathrm{X}-\mathrm{C}$ IIII $\left.{ }^{\mathrm{xx}} \mathrm{XIIII} \mathrm{v}^{\mathrm{o}}\right)$, ff. $139 \mathrm{r}^{\mathrm{o}}-141 \mathrm{r}^{\mathrm{o}}$ (CC IIII ${ }^{\mathrm{xx}} \mathrm{I}-\mathrm{CC}$ IIII ${ }^{\mathrm{xx}}$ III), fol. $152 \mathrm{v}^{\mathrm{o}}\left(\mathrm{CC}\right.$ IIII $\left.^{\mathrm{xx}} \mathrm{XV} \mathrm{v}^{\mathrm{o}}\right)$. 
de la prière et de la messe. L'exemple de la messe instituée par Clément VI contre la peste ${ }^{117}$ est, sur ce point, assez parlant :

Messe pour esciivet mortalité118, lequelle pappes Clemens fist et ordena en college avoech les signeurs cardinaulz et ordena a tous oans ou disans le dite messe II ${ }^{c}$ et LX jours de vrai pardon, et chil qui oent le dite messe doivent porter en leur main une candelle ardant par $V$ jours continueulz ensievans et mors soubite ne leur poura nuire; chou e[s]t cose certaine et approuvee en Avignon et ens es parties d'entour ${ }^{119}$.

La crainte de mourir déconfès et, en corollaire, la volonté de connaître le jour de sa mort, conduisent à une sorte d'art divinatoire porté par la prière : ainsi il est promis à ceux qui diront telle oraison à la Vierge pendant trente jours que il ly sera denonchiet le jour de sen trespas ${ }^{120}$. Pour une autre prière à prononcer à la messe, il est encore précisé : Quiconques dira l'orison a genoulz a le elevation ou en le presence du corps Nostre Signeur par devotion, confes et repentans ne morra point sans confession et se li fera Nostre Seigneur sçavoir l'eure de sen trespas trois jours au devant ${ }^{121}$.

Pour conclure, il faut insister sur le fait que cette présentation est loin d'être parfaite. L'étude du ms. nouv. acq. fr. 4412 est un chantier en cours et il sera nécessaire d'entrer plus avant dans les éléments qui le composent pour le comprendre. Quoi qu'il en soit ce livre de prière de Robert et de Marguerite, seigneur et dame de Wavrin, est un document exceptionnel pour de multiples raisons : ne revenons pas sur le fait qu'on en connaît l'origine et le concepteur (le tournaisien Jean Semont). Insistons davantage sur ce que ce manuscrit peut nous apprendre de la piété d'un grand seigneur et d'une grande dame de la fin du Moyen Âge. On a un témoignage parfait de la place qu'y occupe notamment le culte des saints patrons. Ensuite, l'entourage clérical : c'est un chapelain, un confesseur, peut-être franciscain, qui a conçu et réalisé cet ensemble de textes, partie en latin, partie en « roman », comme il le dit lui-même. On peut encore souligner le fait que ce manuscrit contient un guide pratique de vie religieuse une sorte de mode d'utilisation de la prière et de

117 J. VIARD, La messe pour la peste, dans Bibliothèque de l'École des chartes, t. 61, 1900, pp. 334-338.

118 Ce titre est la traduction littérale de l'introduction du texte édité par VIARD (op. cit., p. 336), seuls manquent le numéro du pape et le millésime : Missa pro evitanda mortalitate, quam dominus papa Clemens sextus fecit et constituit in collegio, cum dominis cardinalibus, anno Domini millesimo CCC XLVIII. Et concessit omnibus predictam missam audientibus et dicentibus ducentas sexaginta dies indulgentie. Et omnes audientes predictam missam debent portare in manu unam candelam ardentem per quinque dies continue sequentes; et eis mors subitanea nocere non poterit. Hoc est certum et approbatum in Avinione et in partibus Avinionensibus.

119 Ibid., ff. $96 \mathrm{r}^{\circ}-101 \mathrm{r}^{\circ}$ (CC XXXVI-CC XLI).

120 Ibid., ff. $187 \mathrm{v}^{0}-191 \mathrm{v}^{0}(\mathrm{CCC}$ XXXIII vo-CCC XXXVII vo $)$.

121 Ibid., ff. $139 \mathrm{r}^{\mathrm{o}}-141 \mathrm{r}^{\mathrm{o}}\left(\mathrm{CC}\right.$ IIII ${ }^{\mathrm{xx}} \mathrm{I}-\mathrm{CC}$ IIII ${ }^{\mathrm{xx}}$ III). 
la messe à l'usage d'un grand laïc. Prières et messes devant accompagner tous les moments de la vie du fidèle. Il nous montre aussi qu'à certain moment la croyance en la vertu bienfaisante de la prière confine à l'utilitarisme et à la superstition, mais que cela ne semble pas inconciliable avec des manifestations de piété conformes aux normes cléricales. On passe de la prière de dévotion à la prière propitiatoire et de là, éventuellement, à des pratiques protectrices ou curatives moins orthodoxes (elles sont toutefois rares).

Mais en tout état de cause, l'influence de l'encadrement clérical se ressent à travers certains traits et certaines exigences caractéristiques : l'assistance à la messe, l'intériorisation des prières, la fréquentation des sacrements (confession et communion), la pratique du jeûne, la nécessaire sincérité de la foi, de la repentance.

Il y a là, sans conteste, une remarquable rencontre entre culture religieuse des clercs et culture religieuse des laïcs. 